\title{
RESEARCH AND APPLICATIONS OF MECHATRONICS TECHNOLOGIES IN MEDICINE
}

\author{
MING-YIH LEE \\ Graduate Institute of Mechanical Engineering, Chang Gung University, Taoyuan, Taiwan
}

\begin{abstract}
Following the promotion of living quality and the implementation of the national health instrumentation program in Taiwan, the demand of research and development for localized medical modalities has become critical. With corporation with Chang Gung Memorial Hospital, the Medical Automation and Rehabilitation Engineering Research Center (MAREC) in Chang Gung University has been dedicated itself to the development of pivotal technologies for medical mechatronics systems I modalities and perform clinical evaluation of newly developed modalities. In this paper, part of the localized medical mechatronics systems and rehabilitative modalities developed in Chang Gung University (Taiwan) was reported.
\end{abstract}

Biomed Eng Appl Basis Comm, 2001 (June); 13: 125-132.

\section{INTRODUCTION}

The number of disabled people in Taiwan is estimated about half million. To prevent the disabled person becomes handicap depends on rehabilitation. The largest cost confronting these individuals is not only medical care, but maintenance, attendant care, home care expense, the loss of productivity because of the inability to work, and the lost wages of the family members who have to take time off from their job in order to help. In addition, due to the fact that decreasing number of child in average family and extending life of average Chinese people, the percentage of elderly person has rapid growing in recent years. According to the government statistics, Taiwan has become an aging society since 1993. However, the significant change of the infrastructure of medical industry is that increase number of elderly patients requiring long-term care and rchabilitation. Thereforc, the needs for developing medical modalities

Received: Jan. 31, 2001; accepted: March 5, 2001

Correspondence: Ming-Yih Lee, Professor,

Dept. of Mechanical Engineering, Chang Gung University, Taoyuan, Taiwan

E-mail: leemiy@mail.cgu.edu.tw in the area of rehabilitation and chronic care are urgent.

In Taiwan, $90 \%$ of medical modalities are imported from aboard, which accounts for annual worth of more than 10 billion NT dollars. The market growth rate is approximately $15 \%$ on average. Following the promotion of living quality and the implementation of the national health instrumentation program in Taiwan, the demand of research and development for localized medical modalities has become critical. In addition, the existing domestic companies manufacturing medical modalities are mostly small in size and lacking independent $R \& D$ capability. The government has set forth the medical modality industry as one of the ten focus blooming industry in Taiwan. The research and development for domestic medical modality and establish "nationalized industry" has brought many attention from industry and academy.

With corporation with Chang Gung Memorial Hospital, the Medical Automation and Rehabilitation Engineering Research Center (MAREC) in Chang Gung University has been dedicated itself to the development of pivotal technologies for medical devices and perform clinical evaluation of newly developed modalities. By considering localized medical device need to fit the physical build of Chinese people and to design man-machine interface in Chincse, we have developed various medical devices for clinical applications. The related technologies developed 
from research projects are also been continuously transferred to local manufacturers for commercialization. Further in conjunction with other local universities, MAREC has been undersigned by the ministry of Education (Taiwan) for establishing the strategic teaching resource center in the area of "Medical Mechatronics". In this paper, accomplishments from several research projects and part of the localized rehabilitative modalities and technical aids developed in Chang Gung University was reported.

\section{Cervical Traction Rehabilitative Modality with EMG Biofeedback}

The intermittent cervical traction is a popular physical therapeutic device for relieving neck pain or cervical radiculopathy [1-3]. There are many different models of commercial therapeutic traction modalities. However, most traction modalities require manual adjustment of traction force and timing control of traction pull/release phases. The traction force and pull/release sequence are set and fixed during traction session. The drawbacks of these traction machines are: (1) Traction force and pull/release phase control are based on open loop control structure. Therefore, muscle injury, neck soreness or pain after traction may occur due to improper traction angle or muscle spasm during traction. (2) Traction force is set based on the subjective judgment and personal experience of the therapist. (3) There are no objective and quantitative measures for assessment of adequate fixed traction force during the traction process.

To reduce the above mentioned drawbacks of conventional traction, modalities, this study is to design a new design of clinical traction therapeutic modality (Figure 1) with closed loop traction force control based on on-line detected EMG signals from paraspinal muscle for relieving neck pain and ceverical radiculopathy in rehabilitation [4]. This traction modality comprises a main controller for control of traction force and a single channel high signal-to-noise ratio EMG scanner with signal processing unit for monitoring and processing EMG signals. The main controller and the EMG scanner is connected through a main $\mathrm{A} / \mathrm{D}$ interface for muscle relaxation assessment and optimal traction force determination. The main controller is also connected to traction actuator through a traction force control interface for adaptive traction force adjustment. This system capable of integrating the EMG biofeedback, closed loop traction force control software and audio-visual alarm signals as one single operable unit to perform the functions of clinical quantitative diagnostics, muscle activity monitoring, EMG biofeedback, muscle relaxation assessment and traction therapy.

From clinical trial in Chang Gung Hospital, the newly designed cervical traction device with real-time traction weight adaptive control based on EMG bio-

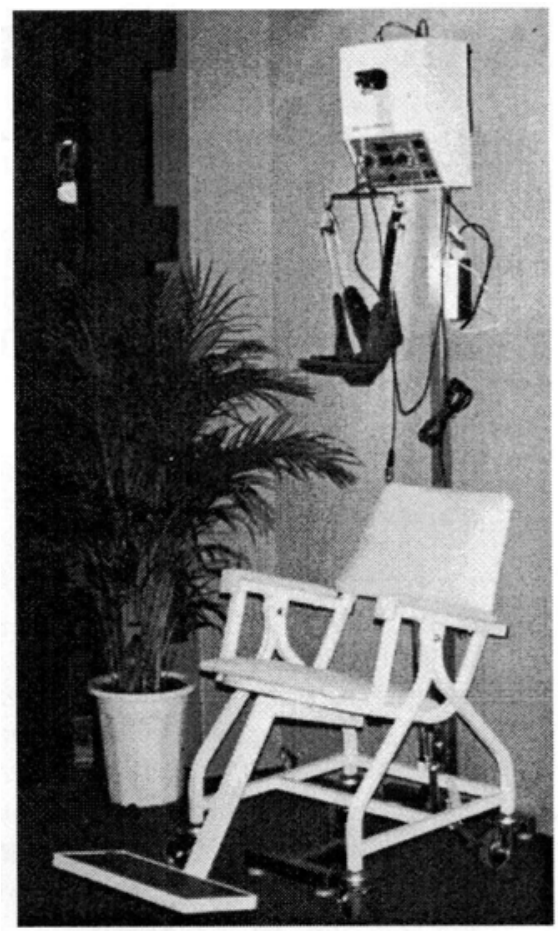

Figure 1 EMG Biofeedback Traction Modality

feedback was found to be more effective in treating patient with neck pain. The results indicated that the use of intermittent cervical traction in sitting position could produce cumulative effect on paraspinal muscle relaxation. The effect of paraspinal muscle relaxation over 7-weeks treatment duration using biofeedback traction device was better than that using conventional protocol.

\section{Labor-Saving Caring Facilities for Patients with Persistent Vegetative State}

In order to reduce the workload and laboring of the nursing staff from long term care of the patients with persistent vegetative state (PPVS), a centralized open care system, which can accommodate 120 PPVS was developed in Chang Gung Memorial Hospital [5]. This system contains a multi-functional patient bed, auto-bathing machine transported by an automatic guided vehicle, new dental cleansing, remote excreta detection diaper, and centralized feeding system.

The multi-functional patient bed consists of a modified bed top with automatic controlled moving bars covered by plastic foam to reduce pressure on the back (Figure $2 a$ ). The inclination of the bed could be adjusted automatically. The patient can be transferred to the auto-bathing shower machine through a robotic arm mounted on the unmanned cart (TRANSCAR). TRANSCAR is an automatic guided vehicle, which travels in a fix route along the bedside with the auto- 


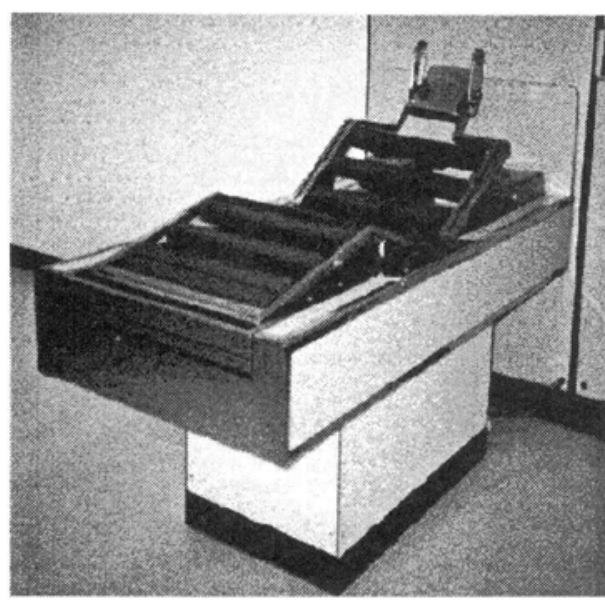

(a)

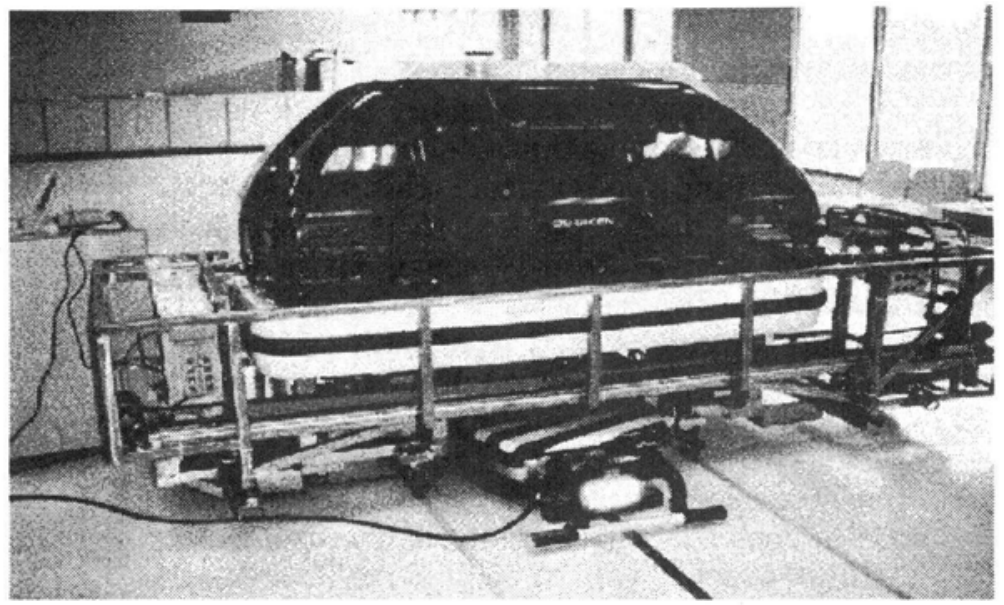

(b)

Figure 2 multi-functional patient bed (a) and auto-bathing shower machine (b)

bathing shower machine on top and stop at the preprogrammed position (Figure $2 b$ ).

The clothing of the PPVS is modified to a single piece with self-adhesive velcro. The T-shaped diaper contains a sensor and alarm for the presence of excreta. All patients' temperature is monitored by an infrared aural thermometry. An automatic jet-spray and suction device for dental cleansing is also designed. Feeding is delivered by an automatic feeder attached with a mini-refrigerating device with patient centralized control system. Clinical trial of this system with 3 healthy young adults and 3 PPVS had been performed. The results showed that the system could reduce the total nursing hours of each PPVS from 4.60 to 1.83 hours/day.

\section{Standing Balance Training System with EMG Biofeedback}

For patients with neurological damage of the central nervous system, such as that due to cerebrovascular accident (CVA), standing balance training is a critical therapeutic procedure to be undertaken beforc walking and self-care training. Conventional static standing steadiness training is mostly done through instructions by a therapist or with a simple standing table. The conventional standing is too simple in design and lacks a balance monitoring function. These drawbacks make standing balance training not only a very time consuming and labor intensive procedure but also incfficient due to lack of self participation or quantitative assessment. This study is to devclop a static standing balance training device with weight bearing biofeedback for rehabilitation.

This standing balance training device (Figure 3) consists of foot pressure sensing instrument for weight bearing measurement, a postural correction mirror for upper extremity postural training, real-time visual weight bearing display, a hand suspension and hip fixation system, a hydraulic height adjustable worktable and interchangeable hand exercise boards. In addition, an auditory system was included to give instruction for standing steadiness training and an alarm signal [6]. Clinical trail of sixty patients with hemiplegia from acute stroke for a period of four weeks are performed. A quantitative evaluation protocol of bilateral asymmetries in weight distribution and postural sway to characterize standing balance was proposed. The experimental results show that with this new standing training device, the static standing steadiness can be trained effectively through weight bearing biofeedback and postural correction mirror in the clinical and home caring environments.

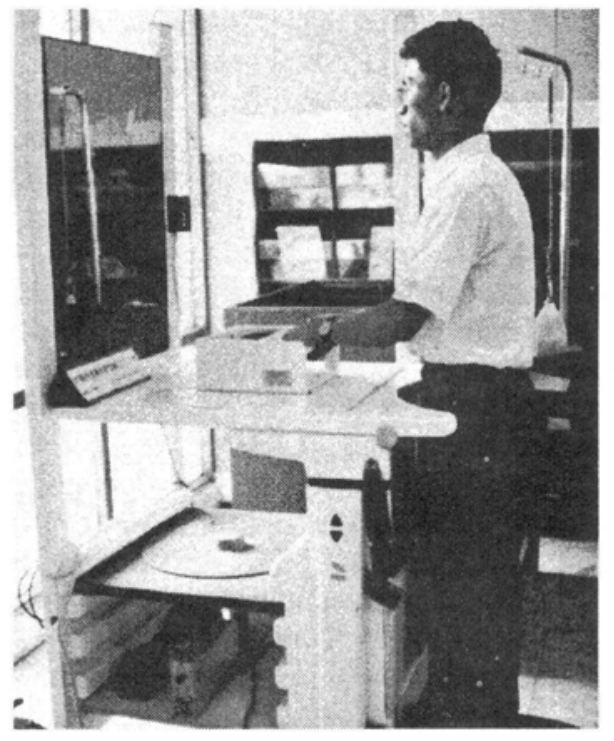

Figure 3 Static standing balance training device 


\section{Dynamic Postural Control Assessment and Training System}

Balance is a complex motor control task, requiring integration of sensory information, neural processing, and biomechanical interaction. This study is to develop a computerized controlled static / dynamic postural control assessment and training device with biofeedback [7]. The hardware design of the proposed dynamic postural control assessment system is shown in Figure 4. The function of the major components of this assessment modality is described below:

(a) Main controller: the controller of this modality is an IBM compatible microcomputer, consisting of $8 \mathrm{MHz} 80486 \mathrm{CPU}, 1024$ Kbytes RAM, a 350 Mbytes hard disk, a 1.2 Mbytes floppy disk for archival and semi-permanent data storage. The keyboard provides a means of inputting textual data into the computer via a standard interface. A PC compatible mouse (Logitech Corporation) allows the user to quickly point to various items (text within menu selections, critical points on graphs) under software control.

(b) $\mathrm{A} / \mathrm{D}$ converter: this allows the user to convert continuous analog signal to digital form. This A/D converter interface card (Advantech Corporation, Model No. PCL-818L) mounted inside the main controller has 16 channel, single ended, +30 V, 12 bits (4096 levels) capability. Inputs of foot-ground reaction forces provided in voltage form by the instrumented platform are channeled through this device. These data were acquired at software selectable rates (maximum of $\mathbf{4 0}$ $\mathrm{MHz}$.

(c) Instrumented force sensing platform: a calibrated foot pressure detection instrumented platform was designed. This platform consists of two separated footpads size $35 \mathrm{~cm} \times 18 \mathrm{~cm}$, with horizontal and vertical grid lines for foot coordinate identification. Four $50 \mathrm{~kg}$ grade strain gage type load cells were mounted in each corner underneath the footpad for sensing normal contact force for each foot. These eight signals evoked by load cells were fed under the platform via a 5meter cable to separate charge amplifiers with a sampling rate of $250 \mathrm{~Hz}$. These signals were then sent through a PC-818L A/D converter for the computer to calculate the ground reaction forces applied to the platform in forward-back $(\mathrm{A} / \mathrm{P})$, and right-left $(\mathrm{M} / \mathrm{L})$ directions. Also the point of force application of the resultant ground reaction forces (i.e. center of pressure, COP) for each feet and the whole body were computed.

(d) Real-time visual feedback display: a menu driven postural control software written in Microsoft $\mathrm{C}$ developed in Window operating environment was integrated into this modality for real-time bio-

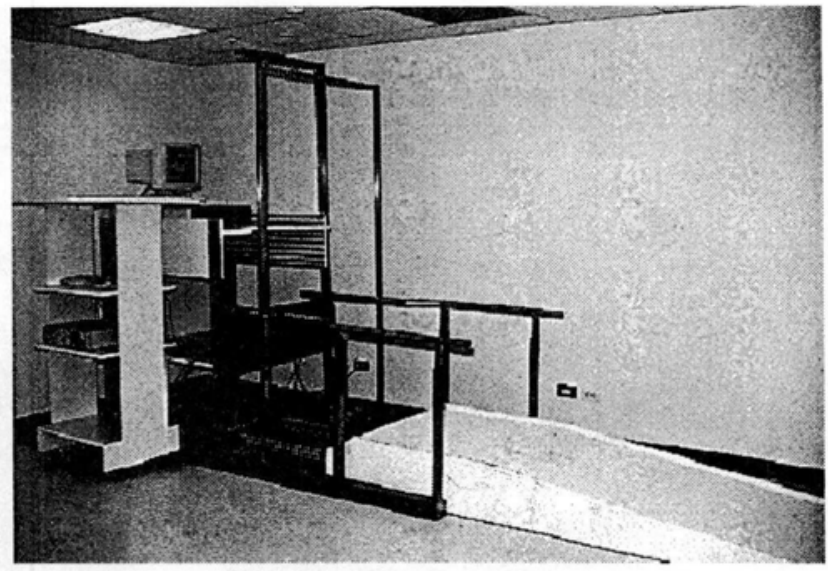

Figure 4 Dynamic postural control assessment and training system

feedback.

(e) Dynamic electromyography: a sixteen channel dynamic EMG system (Myosystem model 2000, Noraxon, USA) provides twelve bioamplification units for the measurement of muscle electromyography signal (raw EMG). The output is bipolar \pm 2.5 volts, input voltage is 12 volts@1.5 a, sensitivity is $1 \mu \mathrm{v}$, amplification bandwidth 15 to $500 \mathrm{~Hz}$, input impedance 10 Mega Ohms.

(f) Range of motion sensor: an electronic inclinometer (NoroTrack 360, Noromed USA Inc) used for knee joint angular displacement measurement was also included in this system. The resolution is \pm 0.1 degree.

Clinical study was undertaken using newly developed assessment system to explore supportive laboratory evidence for clinical observations that a stroke patient has lost functional mobility/ locomotion capability based on dynamic balance responses (Center of Pressure, COP sway patterns) and motor control activities (EMG patterns) during the motor task of sit-tostand (STS). Fourteen stroke patients with right hemiplegia and nine healthy elderly were recruited as the experimental and control groups respectively. Results show that both the motor control patterns and dynamic balance indices correlated well to the extent of mobility impairment evaluated using the traditional FIM method. The important implication from preliminary clinical study for rehabilitation medicine is that the functional mobility capability of stroke patients may be quantified analytically using dynamic balance indices and visualized graphically through EMG motor patterns using our computerized postural control assessment system.

Passive and Active Body Turning Balance As-
sessment and Training Modality
One of the most common activities of daily life is 


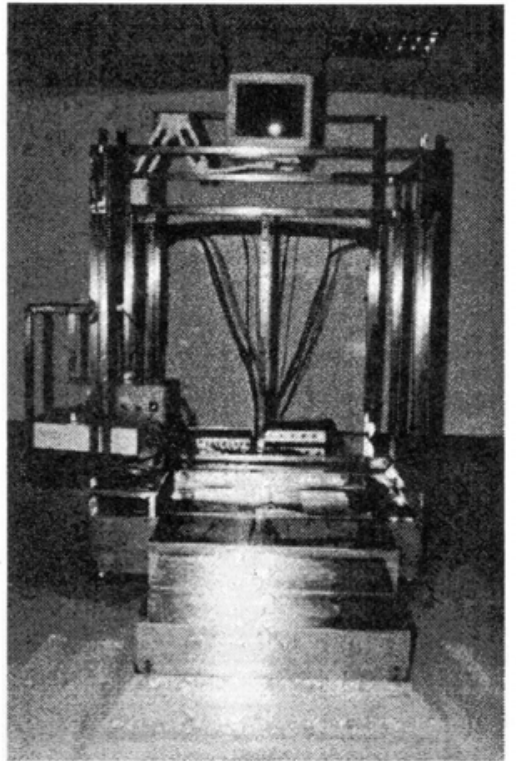

Figure 5 Body turning balance assessment and training modality

to turning body for functional activities, especially for some wheelchair-bounded patients. These people need to turn his body when transfer from bedside to wheclchair; when finish brushing teeth; when leave the breakfast table; after finishing personal hygiene or taking bath; and when transfer from seats on buses, trains, or subways. In short, people are constantly turning their body as they carry out their daily activities, especially for a hemiplegia patient. In a therapeutic setting, one of the most important tasks to train patient for ADL is how to turning body safely for normal functional tasks. Therefore, it is necessary to have an effective tool for assessment and train the patient. In this study, a new passive and active body turning balance assessment and training modality was designed and used for rehabilitation. This training device
(Figure 5) consists of instrumented platform, body turning perturbation input device, foot pressure sensing device, joint angular displacement measuring device, EMG measuring interface, signal processing unit and control software. This newly designed device may be incorporated with static / dynamic postural control training modalities described above as set of quantitative postural stability assessment and training tools for stroke patients.

\section{Portable In-shoe Plantar Pressure Monitoring and Gait Training System}

Impairment of dynamic balance control function is common after stroke, head injury, spinal cord injury, and other conditions of upper motor neuron dysfunction. In such conditions, restricted dynamic balance capabilities markedly decrease their abilities to perform functional activities and increase the risk of fall. Therefore, the assessment and training of dynamic balance control function become the critical steps in rehabilitation program. In order to better understand the biomechanics of the foot and the effectiveness of therapeutic and surgical interventions in the correction and treatment of foot disorders, it is essential to find an objective measure of foot dynamics. Therfore, this research focus on the development of a portable inshoe plantar pressure monitoring and gait training system.

This system consists of three units: a shoe unit, a portable data collection unit, and hand-held computer unit as showed in Figure 6. The shoe unit has twelve thin-film pressure sensors attached to each footpad for plantar pressure detection. The pressure signals are then send and stored in the portable data collection unit. This data collection unit comprises an 8-bit single chip microcomputer, 24-channel A/D converter, 1Mbytes memory, RS 232 port, LCD display, buzzer, and keypad. Finally, the hand-held computer (i.e. PDA) contains application software for sensor calibration, data
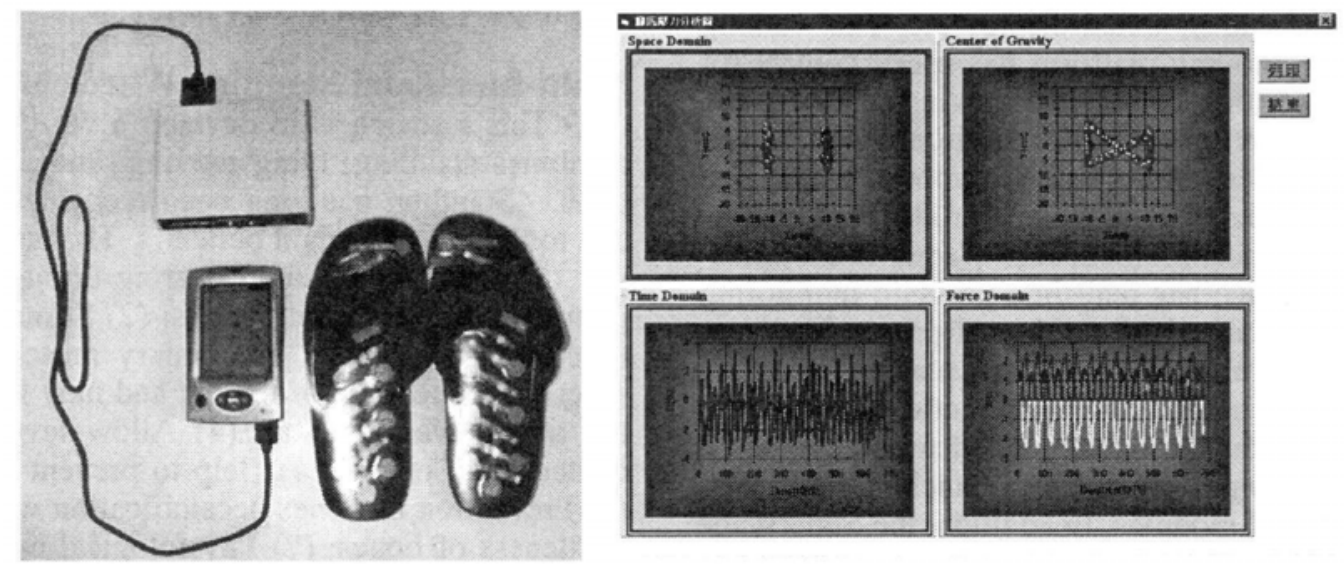

Figure 6 Portable in-shoe plantar pressure monitoring and gait training system 


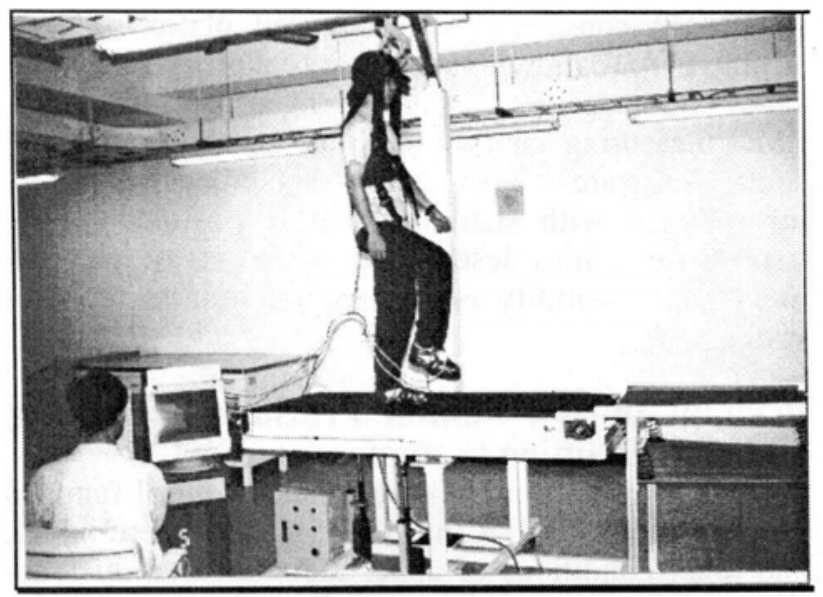

Figure 7 Environmental adaptation system with VR biofeedback technologies

management, data uploading, and plantar pressure analysis. This system capable on integrating dynamic plantar pressure monitoring, audio alarm biofeedback and postural control analysis functions as one single portable device which can be used in hospital and home care rehabilitation for elderly person. It may also be integrated with walking aids for monitoring the dynamic contact pressure of walking aids and foot synchronically for clinical gait training and assessment. Clinical studies in the dynamic balance and gait performance with walking aids for patients with total hip arthroplasty are currently performed.

\section{Environmental Adaptation System with Vir- tual Reality Technologies}

The objective of this research is to develop an environmental adaptation rehabilitation system with virtual reality biofeedback technologies (Figure 7). The hardware of this system consists of walking treadmill, moving platform, body weight support suspension mechanism, wearable foot plantar pressure sensing device, environmental barrier simulated modules, and virtual reality head mounted display etc. The walking treadmill with moving platform has speed control for treadmill belt, tilting control for moving platform and synchronized moving control of environmental barrier simulated modules. Body weight support suspension mechanism utilizes electrical actuator and load cell for body weight support biofeedback. Wearable foot pressure sensing device has real-time pressure monitoring and plantar pressure distribution post-processing functions which may used as a quantitative assessment tool for evaluating rehabilitation therapeutic performance. The environmental barrier simulated modules consist of relay and spring mechanism for stacking and transporting simulated modules. In addition, the Superscape VRT and SDK software were used to design the 3D scene and control interface for visual feedback rcha-
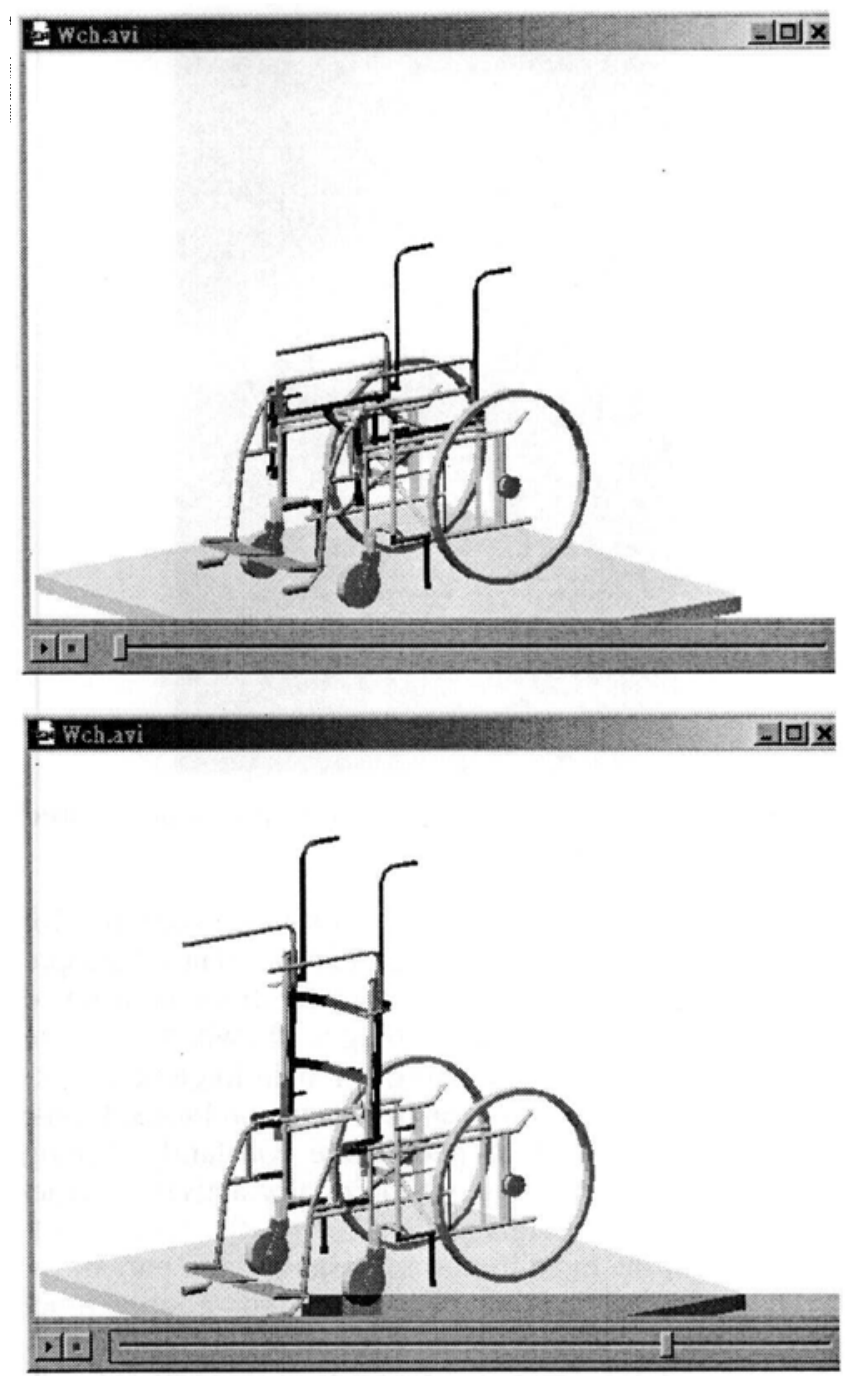

Figure 8 Foldable multi-functional standing wheelchair

bilitation program. This system may be used to train patient to cope with various environmental barriers and thus improving their quality of life.

\section{Multi-functional Standing Wheelchair}

This research is to develop a wheelchair, which combines standing, lying moving, and foldable functions. Standing has long been recognized as beneficial to the non-ambulant person. The benefits including: (1) Encourage weight bearing through bones and joints and prevent contractures; (2) Stimulate postural tone and may control involuntary muscle spasm; (3) Alter the disposition of the gut and may improve bladder and bowel function; (4) Allow level eye-to-eye contact with others; (5) Help to prevent osteoporosis; (6) Prevention of bones decalcification which leads to brittleness of bones; (7) Physiological nutrition of articular cartilage; (8) Prevention of degeneration of internal organs; (9) Prevention of bedsores; (10) In- 

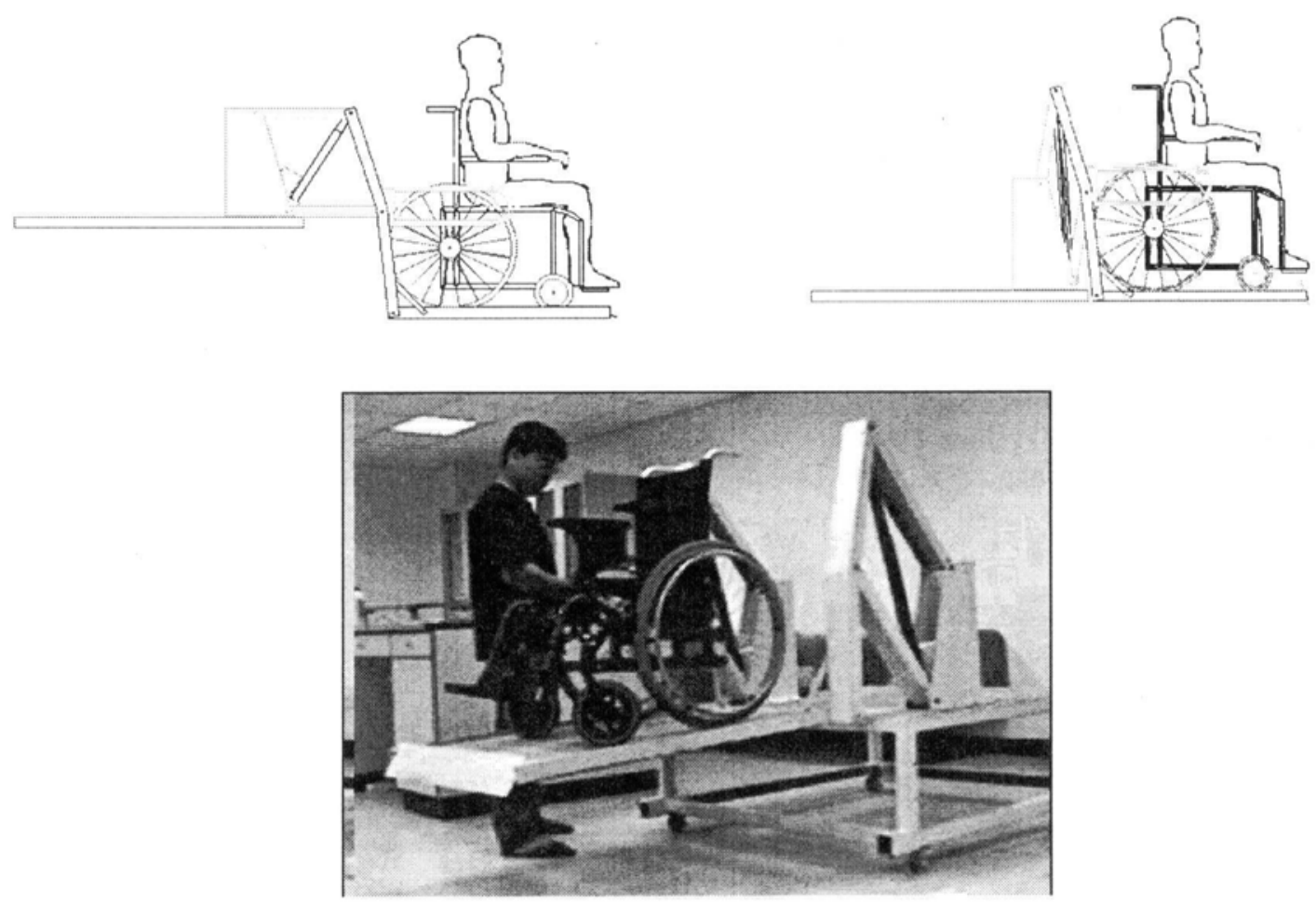

Figure 9 Electrically driven wheelchair lifting device

creasing the injection volume and the minute heart volume; (11) Reduction blood retention in lower limbs vessels; (12) Augmentation of vital capacity, inspiratory and expiratory capacities lungs and diminution of residual capacity; (13) Improvement of elasticity of muscular tissue and ligaments and their nutrishment; (14) Improvement peristaltic movement of bowels and diuresis.

Disabled people with paraplegia or quadriplegia (but with hand function) arc frequently restricted to wheelchairs for mobility. A foldable standing wheelchair (Figure 8) was developed to provide disabled with postural transfer ability from sitting position to standing positions. The basic structure of this newly designed standing wheelchair consist of parallel four bar linkages, armrest, height adjustable footrest, brake mechanisms and front/side stability supports. This wheelchair not only can increase mobility but also may be used to train patients for anti-gravity adaptation.

\section{Electrically Driven Wheelchair Lifting Device}

This study is to develop an electrically driven wheelchair-lifting device, which is fixed inside of fullsize van. A wheelchair user and his wheelchair can be transferred between the ground and the vehicle safely, automatically, and all by himself. The developing process included mechanism conceptual design, simulation, analyzing, implementation and testing. This de- vice may overcome poor-designed barrier-free environment, improve independence of the disabled, and decrease loading of the carcgiver. The prototype was designed and assembled as showed in Figure 9.

\section{CONCLUSION}

The research of applying mechtronics technologies in rehabilitation engineering has been in existence since World War II. With the passage of legislation in Taiwan, it stimulate many research activities in medical mechatronics and rehabilitation engineering in recent years. In this paper, several localized medical mechatronics systems and rehabilitative modalities developed in Chang Gung University was reported. With corporation with Chang Gung Memorial Hospital, the lack of walls around disciplines allows us the freedom to apply engineering theories in solving clinical problems. It is our mission that we will always be an academic engineer standing in the clinical ground to "enable the disabled".

\section{REFERENCES}

1. Nanno, M.," Effects of Intermittent Cervical Traction on Muscle Pain - Flowmetric and Eletromyog- 
raphic Studies of the Cervical traction Paraspinal Muscle", Nippon Ika Duigaka Zusshi, Vol.61,2, pp. 137-147, 1994.

2. Caldwell, J.W., Krusen, E.M., “ Effectiveness of Cervical Traction in Treatment of Neck Problem: Evaluation of Various Methods," Arch Phys Med Rehabil, Vol.43, p.214, 1962.

3. Saunders, H.D.," Use of Spinal Traction in the Treatment of Neck and Back Conditions," Clin Orthop, Vol.179, p.31, 1983.

4. Lee, M.Y., Wong, M.K., Tang, F.T., et.al.," Cervical Traction using EMG Biofeedback," IEEE Eng in Med and Bio, pp.83-87, May/June 1996.

5. Wong, M.K., Lee, M.Y., Tang, F.T.,"The Devel- opment and Clinical trial of Labor - Saving Care Facilities for Patients in a Persistent Vegetative State," The Chang Gung Medical Journal, Vol.20, No.4, pp.272-279, December 1997.

6. Lee, M.Y., Wong, M.K., Tang, F.T.,"Using Biofeedback for Standing Steadiness, Weight Bearing Training", IEEE Eng in Med and Bio, November December 1996, Vol.15, No.6, pp.112-116.

7. Lce, M.Y., Wong, M.K., Tang, F.T., Cheng, P.T., Chiou, W.K. .Lin, P.S, "Comparison of Balance Responses and Motor Pattems during Sit-to-Stand Task with Functional Mobility in the Stroke Patients," Am J of Phys Med and Rehabil, Vol.76, No.5, pp.401-410, September/October 1997. 